\title{
Supernova Explosions and Neutron Star Formation
}

\author{
Hans-Thomas Janka, Konstantinos Kifonidis, and Markus Rampp \\ Max-Planck-Institut für Astrophysik, Karl-Schwarzschild-Str. 1, D-85741 Garching, \\ Germany
}

\begin{abstract}
The current picture of the collapse and explosion of massive stars and the formation of neutron stars is reviewed. According to the favored scenario, however by no means proven and undisputed, neutrinos deposit the energy of the explosion in the stellar medium which surrounds the nascent neutron star. Observations, in particular of Supernova 1987A, suggest that mixing processes play an important role in the expanding star, and multi-dimensional simulations show that these are linked to convective instabilities in the immediate vicinity of the neutron star. Convectively enhanced energy transport inside the neutron star can have important consequences for the neutrino emission and thus the neutrino-heating mechanism. This also holds for a suppression of the neutrino interactions at nuclear densities. Multi-dimensional hydrodynamics, general relativity, and a better understanding of the neutrino interactions in neutron star matter may be crucial to resolve the problem that state-of-the-art spherical models do not yield explosions even with a very accurate treatment of neutrino transport by solving the Boltzmann equation.
\end{abstract}

\section{Introduction}

Baade and Zwicky [2] were the first who speculated about a connection between supernova explosions and the origin of neutron stars. They recognized that stellar cores must become unstable because neutrons, being produced by captures of degenerate electrons on protons, define an energetically advantageous state. The gravitational binding energy liberated by the collapse of a stellar core could power a supernova explosion. More than thirty years later, Colgate and White explored this idea by performing numerical simulations 21]. Their models showed that the prompt hydrodynamical shock, which forms at the moment of core bounce, is not able to reach the outer layers of the star due to severe energy losses by photointegration of iron nuclei. Therefore they suggested that the neutrinos, which are emitted in huge numbers from the hot, collapsed core and carry away the binding energy of the forming neutron star, deposit a fraction of their energy in the stellar medium external to the shock. Later, more accurate simulations with an improved treatment of the input physics such as nuclear equation of state and neutrino transport [9, 10, 11, 12, 49, 3. 60] confirmed the failure of the prompt shock, but could not find the neutrino-driven explosion imagined by Colgate and White.

The discovery of weak neutral currents, whose existence has been predicted by the standard model of electro-weak interactions, had a major influence also on supernova models. It became clear that the neutrinos are strongly coupled to the 
stellar plasma and can escape only on timescales of seconds. This implied that the neutrino luminosities did not become large enough that the rate of energy or momentum transfer by neutrino interactions with the nuclei in the stellar plasma ahead of the supernova shock could become dynamically important. In 1982, however, Jim Wilson discovered 63 that the stalled prompt shock can be revived by neutrino heating in the dissociated postshock medium on a longer timescale. While immediately after shock breakout neutrino emission extracts energy from the shocked gas, energy transfer from neutrinos to the postshock medium is favored hundreds of milliseconds later. If the shock has expanded to a larger radius and the postshock temperature has decreased, energetic neutrinos, which stream up from deeper regions, can deposit a small fraction of their energy in a gain layer behind the shock [8]. Although the efficiency is much lower than imagined by Colgate and White - typically only a few per cent of the neutrino energy are left in the stellar medium - the neutrino heating mechanism can yield the energy of about $10^{51} \mathrm{erg}$ for the explosion of a massive star like, e.g., Supernova 1987A.

Although Wilson's simulations showed the principle viability of this mechanism and theoretical investigations enlightened the underlying physics [4, [, $6,7,7]$, later simulations revealed a strong sensitivity to the details of the post-bounce evolution of the collapsed stellar core 66, 3, 33, 45. General relativity, the nuclear equation of state and corresponding properties of the nascent neutron star, the treatment of the neutrino transport and neutrino-matter interactions, and the structure of the collapsing star have important influence. In particular, later models of Mayle and Wilson [64,65] produced sufficiently powerful explosions only in case of enhanced neutrino emission from the nascent neutron star due to neutron-finger instabilities. Whether such mixing processes take place, however, is not clear to date [15]. Alternatively, the hot neutron star might develop Ledoux convection [22,17] due to negative gradients of lepton number and/or entropy, a possibility which is suggested by the structure obtained in state-of-the-art spherical models for the neutrino-cooling phase of proto-neutron stars [50,48, and by two-dimensional hydrodynamical simulations [38], but is not generally accepted 44 .

Supernova 1987A has provided us with evidence for large-scale mixing processes, which involve the layers in the near vicinity of the neutron star where radioactive nuclei are produced. Indeed, multi-dimensional simulations have shown the existence of hydrodynamical instabilities in the neutrino-heated region already during the first second of the explosion 28, 47, 32, 33, 20. This convective overturn behind the shock was recognized to allow for an explosion even when spherical models do not explode. Also, it enhances the explosion energy and sets the timescale until the shock gains momentum. But still it is not clear whether it is sufficient and crucial for the neutrino-driven mechanism to be at work, or whether it is just an unavoidable side-effect [45]. The existence of these mixing processes, however, definitely means that the events leading to an explosion, the energetics of the explosion, the nucleosynthesis of radioactive elements dur- 
ing the first second, and the composition and distribution of the ejecta can be understood quantitatively only by multi-dimensional simulations.

Unfortunately, the 24 neutrinos from Supernova 1987A, which were recorded in the underground experiments of Kamioka, IMB and Baksan [1], did not provide enough statistical information to draw conclusions on the events which instigated the explosion of the star. Although these neutrinos confirm the basic picture of stellar core collapse and neutron star formation, they are not suitable to support the neutrino-driven mechanism. Therefore the latter may be considered as the currently most favorable explanation for the explosion, but empirical evidence cannot be put forward and numerical models do not draw a clear and unambiguous picture.

It cannot be excluded that the energy for supernova explosions of massive stars is provided by some other mechanism than neutrino heating, for example by magneto-hydrodynamical processes. However, we know that neutrinos with the expected characteristics are emitted from collapsed stellar cores, we know that these neutrinos carry away the gravitational binding energy of the nascent neutron star, we know that neutrino heating must occur behind the stalled shock some time after core bounce, we know that analytic studies and numerical simulations find explosions for a suitable combination of conditions, we know that the energy transfer by neutrinos can be strong enough to account for "normal" explosions with a canonical energy of $\sim 10^{51} \mathrm{erg}$, and we know that the timescale of shock rejuvenation by neutrino heating determines the mass cut such that accretion of the collapsed stellar core and later fallback lead to neutron star masses and supernova nucleosynthesis in rough agreement with observations [23,25.67. Of course, the discrepant results of numerical simulations are unsatisfactory, and major problems are nagging: Why do the supposedly best and most advanced spherical models not produce explosions? Can one trust current multidimensional simulations with their greatly simplified and approximate treatment of neutrino transport? Is rotation in neutrino-driven explosions sufficient to explain the observed large asphericities and anisotropies in many supernovae [24]? What is the reason for the kicks by which pulsars are accelerated to average velocities of several hundred $\mathrm{km} / \mathrm{s}$ presumably during the supernova explosion? What powers hyperenergetic supernovae, which seem to release up to 50 times more kinetic energy than ordinary explosions of massive stars [30]?

This paper gives an overview over the major lines of research on the standard explosion scenario of massive stars, and on neutron star formation, where progress has been achieved during the past few years or will be coming up in the near future. In Section 2 the physics of neutrino-driven explosions will be discussed in some detail, and an analytic toy model will be described which allows one a deeper understanding of the requirements of neutrino-driven explosions. In Section 3 the current status of supernova modeling in spherical symmetry will be outlined, with particular focus on results with neutrino transport by solving the Boltzmann equation. In Section 4 the relevance of convective overturn in the neutrino-heated layer will be discussed. New results of two-dimensional simulations will be presented, which take into account the nucleosynthesis during 
Fig. 1. Sketch of the post-collapse stellar core during the neutrino heating and shock revival phase. $R_{\nu}$ is the neutrinosphere radius, $R_{\mathrm{ns}}$ the protoneutron star radius, $R_{\mathrm{g}}$ the gain radius outside of which net neutrino heating exceeds neutrino cooling, and $R_{\mathrm{S}}$ is the shock radius. The shock expansion is impeded by mass infall at a rate $\dot{M}$, but supported by convective energy transport from the region of strongest neutrino heating into the post-shock layer. Convection inside the nascent neutron star raises the neutrino luminosities.

the explosion and follow the shock from the moment of its formation until it breaks out of the surface of the star. In Section 5, two-dimensional hydrodynamical models of the neutrino-cooling phase of newly formed neutron stars will be described. The effects of rotation and of a suppression of neutrino-nucleon interactions by nucleon correlations in the nuclear medium will be addressed. Section 6 will contain a summary and conclusions.

\section{The Explosion Mechanism}

The physics of neutrino-driven explosions is discussed, first on the level of basic considerations, then with the help of an analytic toy model, which allows one to study the competing effects that determine the destiny of the stalled supernova shock.

\subsection{Neutrino Heating}

Figure 1 displays a sketch of the neutrino cooling and heating regions outside the proto-neutron star at the center. The main processes of neutrino energy deposition are the charged-current reactions $\nu_{e}+n \rightarrow p+e^{-}$and $\bar{\nu}_{e}+p \rightarrow n+e^{+}$[8]. With the neutrino luminosity, $L$, the average squared neutrino energy, $\left\langle\epsilon^{2}\right\rangle$, and the mean value of the cosine of the angle $\theta_{\nu}$ of the direction of neutrino propagation relative to the radial direction, $\langle\mu\rangle=\left\langle\cos \theta_{\nu}\right\rangle$, being defined as moments of the neutrino phase space distribution function $f(r, t, \mu, \epsilon)$ by integration over energies $\epsilon$ and angles $\mu$ according to

$$
\begin{aligned}
& L=4 \pi r^{2} \frac{2 \pi c}{(h c)^{3}} \int_{-1}^{+1} \mathrm{~d} \mu \int_{0}^{\infty} \mathrm{d} \epsilon \epsilon^{3} \mu f \\
& \left\langle\epsilon^{2}\right\rangle=\int_{-1}^{+1} \mathrm{~d} \mu \int_{0}^{\infty} \mathrm{d} \epsilon \epsilon^{5} f \cdot\left\{\int_{-1}^{+1} \mathrm{~d} \mu \int_{0}^{\infty} \mathrm{d} \epsilon \epsilon^{3} f\right\}^{-1},
\end{aligned}
$$




$$
\langle\mu\rangle=\int_{-1}^{+1} \mathrm{~d} \mu \int_{0}^{\infty} \mathrm{d} \epsilon \epsilon^{3} \mu f \cdot\left\{\int_{-1}^{+1} \mathrm{~d} \mu \int_{0}^{\infty} \mathrm{d} \epsilon \epsilon^{3} f\right\}^{-1},
$$

the heating rate per nucleon $(N)$ is approximately given by

$$
\begin{aligned}
Q_{\nu}^{+} & \approx 110 \cdot\left(\frac{L_{\nu_{e}, 52}\left\langle\epsilon_{\nu_{e}, 15}^{2}\right\rangle}{r_{7}^{2}\langle\mu\rangle_{\nu_{e}}} Y_{n}+\frac{L_{\bar{\nu}_{e}, 52}\left\langle\epsilon_{\bar{\nu}_{e}, 15}^{2}\right\rangle}{r_{7}^{2}\langle\mu\rangle_{\bar{\nu}_{e}}} Y_{p}\right) \quad\left[\frac{\mathrm{MeV}}{\mathrm{s} \cdot N}\right] \\
& \approx 55 \cdot \frac{L_{\nu, 52}\left\langle\epsilon_{\nu, 15}^{2}\right\rangle}{r_{7}^{2} f}\left[\frac{\mathrm{MeV}}{\mathrm{s} \cdot N}\right]
\end{aligned}
$$

where $Y_{n}$ and $Y_{p}$ are the number fractions of free neutrons and protons (number densities divided by baryon number density), respectively. In the second equation $Y_{n}+Y_{p} \approx 1$, and equal luminosities and spectra for $\nu_{e}$ and $\bar{\nu}_{e}$ were assumed. $L_{\nu, 52}$ denotes the total luminosity of $\nu_{e}$ plus $\bar{\nu}_{e}$ in $10^{52} \mathrm{erg} / \mathrm{s}, r_{7}$ the radial position in $10^{7} \mathrm{~cm},\left\langle\epsilon_{\nu, 15}^{2}\right\rangle$ is measured in units of $15 \mathrm{MeV}$, and $f=\langle\mu\rangle_{\nu}$ is very small in the opaque regime where the neutrinos are isotropic, adopts a value of about 0.25 around the neutrinosphere, and approaches unity for radially streaming neutrinos very far out. Note that the "flux factors" $\langle\mu\rangle_{\nu}$ determines the neutrino energy density at a radius $r$ according to $\varepsilon_{\nu}(r)=L_{\nu} /\left(4 \pi r^{2} c\langle\mu\rangle_{\nu}\right)$.

Using this energy deposition rate, neglecting energy losses due to the reemission of neutrinos, and assuming that the gravitational binding energy of a nucleon in the neutron star potential is (roughly) balanced by the sum of internal and nuclear recombination energies after accretion of the infalling matter through the shock, one can estimate (very approximately) the explosion energy to be of the order

$$
E_{\exp } \sim 10^{51} \cdot \frac{L_{\nu, 52}\left\langle\epsilon_{\nu, 15}^{2}\right\rangle}{R_{\mathrm{g}, 7}^{2} f}\left(\frac{\Delta M}{0.1 M_{\odot}}\right)\left(\frac{\Delta t}{0.1 \mathrm{~s}}\right)-E_{\mathrm{gb}}+E_{\mathrm{nuc}} \quad[\mathrm{erg}] .
$$

Here $\Delta M$ is the heated mass, $\Delta t$ the typical heating timescale, $E_{\mathrm{gb}}$ the (net) total gravitational binding energy of the overlying, outward accelerated stellar layers, and $E_{\text {nuc }}$ the additional energy from explosive nucleosynthesis, which is a significant contribution of a few $10^{50}$ erg only for progenitors with main sequence masses above $20 M_{\odot}$, and which roughly compensates $E_{\mathrm{gb}} 1$.

It is not easy to infer from Eq. (5) the dependence of the explosion energy on the neutrino luminosity. On the one hand,

$$
E_{\exp } \propto Q_{\nu}^{+} V \Delta t \propto \frac{L_{\nu}\left\langle\epsilon_{\nu}^{2}\right\rangle}{R_{\mathrm{g}}^{2}} \Delta M \Delta t \propto L_{\nu} \Delta \tau \Delta t,
$$

\footnotetext{
1 The latter statement is supported by the following argument (S. Woosley, personal communication): Material with a specific gravitational binding energy $\Phi_{\text {grav }}$ which is equal to or larger than the nuclear energy release per gram in Si burning, $e_{\text {nuc }} \sim$ $10^{18} \mathrm{erg} / \mathrm{g}$, is located interior to the radius where the temperatures can become high enough $\left(T \gtrsim 5 \times 10^{9} \mathrm{~K}\right)$ for explosive nucleosynthesis of ${ }^{56} \mathrm{Ni}$. ¿From $\Phi_{\text {grav }}=G M / r \gtrsim$ $10^{18} \mathrm{erg} / \mathrm{g}$ one estimates a radius of $r \lesssim 2 \times 10^{8} \mathrm{~cm}$, and from $\frac{4}{3} \pi r^{3} a T^{4} \sim 10^{51} \mathrm{erg}$ with $T \gtrsim 5 \times 10^{9} \mathrm{~K}$ one finds $r \lesssim 4 \times 10^{8} \mathrm{~cm}$. This means that the energy release from explosive nucleosynthesis is easily able to account for the gravitational binding energy of the burning material.
} 
where $V$ is the heated volume between gain radius and shock, and $\Delta \tau$ the optical depth of the heating layer. On the other hand,

$$
\Delta \tau \propto\left\langle\epsilon_{\nu}^{2}\right\rangle R_{\mathrm{g}} \rho_{\mathrm{g}} \propto\left\langle\epsilon_{\nu}^{2}\right\rangle R_{\mathrm{g}} T_{\mathrm{g}}^{3} \propto L_{\nu}^{1 / 2}\left\langle\epsilon_{\nu}^{2}\right\rangle^{3 / 2}
$$

Here $\rho \propto T^{3}$ was assumed for the relation between density and temperature in the heating layer [7], and $Q_{\nu}^{+}\left(R_{\mathrm{g}}\right)=Q_{\nu}^{-}\left(R_{\mathrm{g}}\right)$ was used at the gain radius, where neutrino heating is balanced by neutrino cooling. The energy loss rate $Q_{\nu}^{-}$by neutrinos produced in capture reactions of nondegenerate electrons and positrons on nucleons, scales with $T^{6}$. Combining Eqs. (6) and (7) yields

$$
E_{\exp } \propto L_{\nu}^{3 / 2}\left\langle\epsilon_{\nu}^{2}\right\rangle^{3 / 2} \Delta t \propto L_{\nu}^{9 / 4} \Delta t
$$

when $\left\langle\epsilon_{\nu}^{2}\right\rangle \propto T_{\nu}^{2} \propto L_{\nu}^{1 / 2}$ is used for black-body like emission.

If the expansion velocity were simply proportional to $E_{\exp }^{1 / 2}$, in which case the time $\Delta t$ for the shock to reach a given radius would be $\Delta t \propto E_{\exp }^{-1 / 2}$, then $E_{\exp } \propto L_{\nu}^{3 / 2}[7]$. However, when shock expansion sets in, most of the energy is internal energy, but not kinetic energy, making the relation $\Delta t \propto E_{\exp }^{-1 / 2}$ very questionable. The actual variation of $\Delta t$ with the inverse of the neutrino luminosity can be steeper.

\subsection{Requirements for Neutrino-Driven Explosions}

In order to get explosions by the delayed neutrino-heating mechanism, certain conditions need to be fulfilled. Expansion of the postshock region requires sufficiently large pressure gradients near the radius $R_{\text {cut }}$ of the developing mass cut. If one neglects self-gravity of the gas in this region and assumes the density profile to be a power law, $\rho(r) \propto r^{-n}$ (which is well justified according to numerical simulations which yield a power law index of $n \approx 3[5]$ ), one gets $P(r) \propto r^{-n-1}$ for the pressure in an atmosphere near hydrostatic equilibrium. Outward acceleration is therefore maintained as long as the following condition for the "critical" internal energy density $\varepsilon$ holds:

$$
\left.\frac{\varepsilon_{\mathrm{c}}}{G M \rho / r}\right|_{R_{\mathrm{cut}}}>\frac{1}{(n+1)(\gamma-1)} \cong \frac{3}{4},
$$

where use was made of the relation $P=(\gamma-1) \varepsilon$. The numerical value was obtained for $\gamma=4 / 3$ and $n=3$. This condition can be converted into a criterion for the entropy per baryon, $s$. Using the thermodynamical relation for the entropy density normalized to the baryon density $n_{b}, s=(\varepsilon+P) /\left(n_{b} T\right)-\sum_{i} \eta_{i} Y_{i}$ where $\eta_{i}\left(i=n, p, e^{-}, e^{+}\right)$are the particle chemical potentials divided by the temperature, and assuming completely disintegrated nuclei behind the shock so that the number fractions of free protons and neutrons are $Y_{p}=Y_{e}$ and $Y_{n}=1-Y_{e}$, respectively, one gets

$$
\left.s_{\mathrm{c}}\left(R_{\mathrm{cut}}\right) \gtrsim 14 \frac{M_{1}}{r_{7} T}\right|_{R_{\mathrm{cut}}}-\left.\ln \left(1.27 \cdot 10^{-3} \frac{\rho_{9} Y_{n}}{T^{3 / 2}}\right)\right|_{R_{\mathrm{cut}}}\left[k_{\mathrm{B}} / N\right] .
$$


In this approximate expression a term with a factor $Y_{e}$ was dropped (its absolute value being usually less than 0.5 in the considered region), nucleons were assumed to obey Boltzmann statistics, and $T$ is measured in $\mathrm{MeV}, M_{1}$ in units of $M_{\odot}$, $\rho_{9}$ in $10^{9} \mathrm{~g} / \mathrm{cm}^{3}$, and $r_{7}$ in $10^{7} \mathrm{~cm}$. Inserting typical numbers $(T \approx 1.5 \mathrm{MeV}$, $\left.Y_{n} \approx 0.3, R_{\text {cut }} \approx 1.5 \cdot 10^{7} \mathrm{~cm}\right)$, one finds $s>15 k_{\mathrm{B}} / N$, which gives an estimate of the entropy in the heating region when expansion is going to take place.

Since the entropy and energy density in the postshock later are raised by neutrino energy deposition, the conditions of Eqs. (9) and (10) imply requirements on the neutrino emission of the proto-neutron star. These can be derived by the following considerations. A stalled shock is converted into a moving one only, when the neutrino heating is strong enough to increase the pressure behind the shock by a sufficient amount. From the Rankine-Hugoniot relations at the shock, a criterion can be deduced for the heating rate per unit mass, $q_{\nu}$, behind the shock, which leads to a positive postshock velocity $\left(u_{1}>0\right)$ [12:

$$
q_{\nu}>\frac{2 \beta-1}{\beta^{3}(\beta-1)(\gamma-1)} \frac{\left|u_{0}\right|^{3}}{\eta R_{\mathrm{s}}} .
$$

Here $\beta$ is the ratio of postshock to preshock density, $\beta=\rho_{1} / \rho_{0}, \gamma$ the adiabatic index of the gas (assumed to be the same in front and behind the shock), and $\eta$ defines the fraction of the shock radius $R_{\mathrm{s}}$ where net heating by neutrino processes occurs: $\eta=\left(R_{\mathrm{s}}-R_{\mathrm{g}}\right) / R_{\mathrm{s}} . u_{0}$ is the preshock velocity, which is a fraction $\alpha$ (analytical and numerical calculations show that typically $\alpha \approx 1 / \sqrt{2}$ ) of the free fall velocity: $u_{0}=\alpha \sqrt{2 G M / r}$. Assuming a strong shock, one has $\beta=(\gamma+1) /(\gamma-1)$, which becomes $\beta=7$ for $\gamma=4 / 3$. With typical numbers, $R_{\mathrm{s}}=100 \mathrm{~km}, M=M_{1}=1 M_{\odot}$, and $\eta \approx 0.4$, one derives for the critical luminosity of $\nu_{e}$ plus $\bar{\nu}_{e}$ :

$$
L_{\nu, 52}\left\langle\epsilon_{\nu, 15}^{2}\right\rangle>4.4 \frac{M_{1}^{3 / 2}}{R_{\mathrm{s}, 7}^{1 / 2}}
$$

Since this discussion was very approximate, e.g., the reemission of neutrinos was ignored and properties depending on the structure of the collapsed stellar core were absorbed into free parameters, the analysis cannot yield a quantitatively meaningful value for the threshold luminosity. However, the existence of a lower bound on the neutrino luminosity as found in numerical simulations [32,33], is confirmed. Above this threshold value, neutrino heating of the gas behind the shock is strong enough to drive an expansion of the gain layer.

\subsection{Analytic Toy Model}

The discussion in the previous section was overly simplified. The behavior of the stagnant shock does not only depend on the neutrino heating in the gain region, but is also influenced by the energy loss and the settling of the cooling layer, and by the mass infall from the collapsing star star ahead of the shock. Only hydrodynamical simulations can determine the shock evolution in response to 

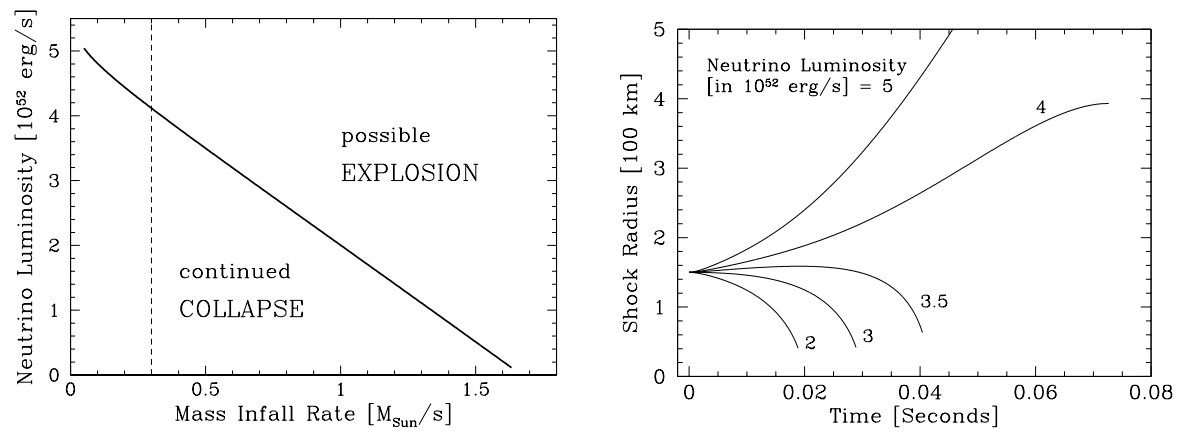

Fig. 2. Left: Phase diagram for successful explosion or continued stellar collapse in dependence upon the rate at which gas falls into the shock and upon the $\nu_{e}$ plus $\bar{\nu}_{e}$ luminosity of the nascent neutron star. The mass of the neutron star was assumed to be $1.25 M_{\odot}$. Right: Shock positions as functions of time for different values of the $\nu_{e}$ plus $\bar{\nu}_{e}$ luminosity of the neutron star (according to the labels) and fixed value of the rate at which gas falls into the shock (marked by the dashed line in the left plot). For neutrino luminosities above the critical value (where the dashed line crosses the solid line in the left plot) explosions can occur.

these different, partly competing effects. Analytic discussions, however, can help one understanding the significance of different effects and thus can supplement more detailed, but less transparent supercomputer calculations.

The conditions in the region of neutrino heating and the details of the heating process were analysed by Bethe and Wilson [8] and Bethe [4, [4, 6].7]. Burrows and Goshy [18] considered the post-bounce accretion phase of the supernova shock as a quasi steady-state situation, and thus replaced the time-dependent partial differential equations of hydrodynamics by a set of ordinary differential equations to determine the radial position of the standing shock by an eigenvalue analysis. While this approach captures an interesting aspect of the problem, it has serious weaknesses. The accretion flow between the shock and the neutron star does not need to be stationary, but neutrino-heated matter may stay in the gain region, or gas will pile up on the forming neutron star when neutrinos are unable to remove energy quickly enough for the gas to settle. In particular, when the shock accelerates outward, the transition from accretion to outflow cannot be described as a stationary situation.

Since the gas falling into the stalled shock is strongly decelerated and the postshock velocities are smaller than the local sound speed, the structure of the collapsed stellar core is rather simple and can be well described by hydrostatic equilibrium. This allows for an approximate treatment, which is complementary to the approach taken by Burrows and Goshy [18]: Integrating the stellar structure over radius leads to conservation laws for the total mass and energy in the gain layer. The time-dependent radius and velocity of the shock can then be obtained as solutions of an initial value problem, which reflects the fact that the destiny of the shock depends on the initial conditions and is controlled by the 
cumulative effects of neutrino energy deposition and mass accumulation in the gain layer 31.

Such an analysis also demonstrates the existence of a threshold value for the neutrino luminosity from the neutron star, which is needed to drive shock expansion. This threshold luminosity depends on the rate, $\dot{M}$, of mass infall to the shock, on the neutron star mass and radius, and to some degree also on the shock stagnation radius. Taking into account only the main dependence on $\dot{M}$, it can roughly be written as [31]

$$
L_{\nu, \mathrm{crit}}(\dot{M}) \approx L_{0}-L_{1}\left(\frac{\dot{M}}{M_{\odot} / \mathrm{s}}\right),
$$

with $L_{0} \approx 5 \times 10^{52} \mathrm{erg} \mathrm{s}^{-1}$ and $L_{1} \approx 3 \times 10^{52} \mathrm{erg} \mathrm{s}^{-1}$ for the conditions of Fig. 2 .

The neutrino heating in the gain layer is not the only important factor that determines the shock propagation. Energy loss by neutrino emission in the cooling layer has a considerable influence, because it regulates the settling of the matter that is accreted by the nascent neutron star, and therefore the advection of gas through the heating layer. If cooling is inefficient, gas piles up on the neutron star and pushes the shock farther out. If cooling is very efficient, the gas contracts quickly and more gas is dragged downward through the gain radius, extracting mass and energy from the gain layer and thus weakening the support for the shock. This also means that the infall velocity behind the shock increases and the timescale for the gas to stay in the gain layer is reduced. Therefore the efficiency of neutrino energy deposition drops. Such an effect is harmful for shock expansion. It can be diminished by higher $\nu_{e}$ and $\bar{\nu}_{e}$ luminosities from the neutrinosphere, which lead to an enhancement of neutrino absorption relative to neutrino emission. On the other hand, muon and tau neutrino and antineutrino production in the accretion layer of the neutron star has a desastrous consequence for the shock, because it is a sink of energy that leaves the star without any significant positive effect above the neutrinosphere, where only $\nu_{e}$ and $\bar{\nu}_{e}$ can be absorbed by free nucleons.

These different, competing processes combined explain the slope of the critical line in the left plot of Fig. 2 and in Eq. (13). Shock expansion and acceleration are easier for high mass infall rates, $\dot{M}$, into the shock and for high $\nu_{e}$ and $\bar{\nu}_{e}$ luminosities from the nascent neutron star. These luminosities need to be larger when $\dot{M}$ is small. It must be pointed out here, however, that this dependence is a consequence of the fact that the temperature in the cooling layer is considered as a parameter of the discussion. It is assumed to be equal to the neutrinospheric temperature and thus to be mainly determined by the interaction with the neutrino flux from the core of the neutron star, but not by the mass infall and the dynamics in the accretion layer.

Emission of muon and taun neutrinos and antineutrinos from the cooling region is not included in the results displayed in Fig. 2. It would move the critical line to appreciably higher values of the $\nu_{e}$ plus $\bar{\nu}_{e}$ luminosity of the collapsed core, which is given along the ordinate. 


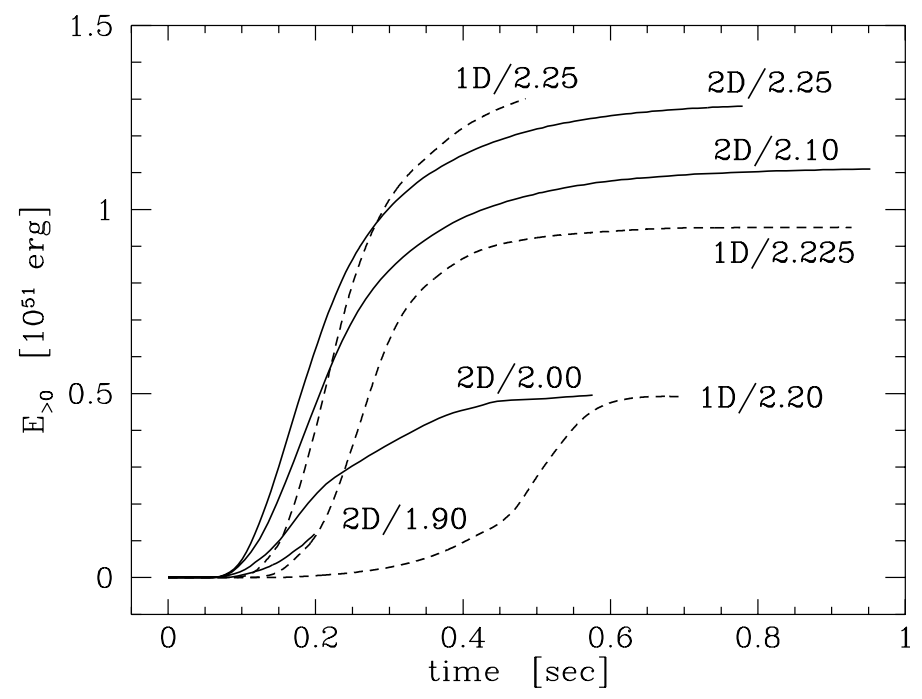

Fig. 3. Explosion energies $E_{>0}(t)$ for spherically symmetric models ("1D", dashed lines) and two-dimensional ("2D") supernova models (solid lines) 32,33. The latter take into account convective overturn between the supernova shock and the neutrinoheating region. The curves display the evolution as a function of time after shock formation for different values of the $\nu_{e}$ luminosity (labeled in units of $10^{52} \mathrm{erg} / \mathrm{s}$ ). The latter was used as a free parameter at the surface of the nascent neutron star and was roughly equal to the $\bar{\nu}_{e}$ luminosity. Below the smallest given luminosities, the considered $15 M_{\odot}$ star does not explode in $1 \mathrm{D}$ and acquires too low an expansion energy in $2 \mathrm{D}$ to unbind the whole stellar mantle and envelope.

Neutrino heating is stronger close to the gain radius than right behind the shock. Using an isentropic profile in the gain layer, the evaluation, however, implies very efficient energy transport, e.g., by convective motions in the gain layer. This enhances the postshock pressure and reduces the loss of energy from the gain layer, which is associated with the inward advection of neutrino-heated gas.

Solutions of the toy model for varied parameters show that the energy in the gain layer and therefore the explosion energy of the supernova is limited to some $10^{51} \mathrm{erg}$. The reason for this is the following. Neutrino energy deposition proceeds by $\nu_{e}$ and $\bar{\nu}_{e}$ absorption on nucleons. The heated gas expands away from the region of strongest heating as soon as the nucleons have absorbed an energy roughly of the order of the gravitational potential energy, with only a small time lag because of the inertia of the shock, which is confined by the ram pressure of the collapsing stellar material. This does not allow the net energy of the heated gas to become very large. Typically it is of the order of $\sim 5 \mathrm{MeV}$ per nucleon. With a mass in the gain layer of several $0.01 M_{\odot}$ up to $\sim 0.1 M_{\odot}$, the total energy does therefore not exceed a few $10^{51} \mathrm{erg}$. 
Figure 3 clearly shows this saturation of the explosion energy, which occurs when the gain layer and the shock are expanding. In this sense, neutrino-driven explosions are "self-regulated": Further energy deposition is quenched as the baryons move out of the region of high neutrino fluxes.

The heating rate increases with the neutrino luminosity and the deposited energy is higher for a larger mass in the gain layer (Eq. 5). However, the expansion timescale during which the gas is exposed to high neutrino fluxes, drops when the heating is stronger. Therefore the explosion energy is extremely sensitive to the neutrino luminosity only around the threshold value for getting an explosion.

Neutrino-driven explosions are likely to be "delayed" (up to a few $100 \mathrm{~ms}$ after core bounce) rather than "late" (after a few seconds). The density between the gain radius and the shock decreases with time because the proto-neutron star contracts and the gas infall to the shock drops rapidly as time goes on. Therefore the mass $\Delta M$ in the heated region shrinks and the shock must recede to a small radius around or even below $100 \mathrm{~km}$, not favoring a later explosion.

Fulfilling the "explosion criterion" of Fig. 2 once is no guarantee for a successful explosion. The push from the heating region has to be maintained until the material that carries the bulk of the energy is moving ballistically, and a fair fraction of this energy has been converted from internal to kinetic energy. Otherwise, if energy losses by neutrino emission or $P \mathrm{~d} V$ on the contracting proto-neutron star start to dominate the energy input by neutrino absorption, the pressure-supported expansion can break down again and re-collapse can occur. However, once shock expansion sets in, the conditions for further neutrino heating improve rapidly, and the optical depth of the growing gain layer to neutrinos increases. Provided the neutrino luminosity does not drop, an explosion becomes unavoidable. This requirement favors a high core neutrino luminosity over accretion luminosity to power neutrino-driven explosions.

\section{Status of Spherical Simulations}

Recently, a major shortcoming of previous supernova models has been removed, at least in spherical models. Instead of treating multi-frequency neutrino transport by a flux-limited diffusion approximation [64,65, 49, 96, the Boltzmann equation can now be solved in connection with hydrodynamical simulations, either by direct discretisation [46] or by a variable Eddington factor technique [54, even in the general relativistic case 42]. For the first time, the numerical deficiencies of the models are therefore smaller than the uncertainties of the input physics.

The more accurate treatment of the transport, in particular in the semitransparent neutrino-decoupling region around and outside of the neutrinosphere up to the shock, favors higher energy transfer to the stellar gas in the cooling layer and in the gain layer 43,69. Nevertheless, the results of these simulations are disappointing. In spherical symmetry, the models do not explode, neither in the Newtonian (Fig. 1), nor in the general relativistic case. 
Fig. 4. Trajectories of mass shells in the core of a collapsing $15 M_{\odot}$ star from a Newtonian simulation with Boltzmann neutrino transport [53,54]. The shells are equidistantly spaced in steps of $0.02 M_{\odot}$. The boundaries of the iron core, silicon shell and neonmagnesium shell are indicated by bold lines. The fat, solid curve rising up at 0.21 seconds after the start of the simulation marks the position of the supernova shock, the dashed line denotes the gain radius. In this spherically symmetric simulation, muon and tau neutrinos and antineutrinos were neglected, which favors an explosion. Nevertheless, the shock recedes after having expanded to more than $300 \mathrm{~km}$.

These current models, however, neglect convective effects inside the nascent neutron star as well as in the neutrino-heated region. Since convection has been recognized to be very important, such simulations do not treat the full supernova problem and do not really allow for conclusions about the viability of the neutrino-driven mechanism. Multi-dimensional simulations with Boltzmann neutrino transport are called for.

\section{Hydrodynamic Instabilities during the Explosion}

During the explosion of a supernova, hydrodynamic instabilities and convective processes can occur on different scales in space and time. Convective motions inside the nascent neutron star can speed up the energy transport and raise the neutrino luminosities during a period of seconds (Section 5). In the neutrinoheated region, convective overturn during the first second of the explosion carries hot matter towards the shock front and brings cool gas into the region of strongest neutrino heating near the gain radius. This has important influence on the start of the explosion and the nucleosynthesis of radioactive elements. When the shock propagates through the mantle and envelope of the disrupted star, Rayleigh-Taylor instabilities destroy the onion-shell structure of the progenitor and mix radioactive material with high velocities from near the neutron star into the helium and even hydrogen shells of the star. In this section, the early postshock convection and its interaction with the hydrodynamic instabilities at the composition interfaces of the progenitor star will be discussed. 
Fig. 5. Inhomogeneous distribution of neutrino-heated, hot gas which rises in mushroom-like bubbles, and cooler gas that is accreted through the supernova shock (bumpy discontinuity at about $3000 \mathrm{~km}$ ) and falls in long, narrows streams towards the newly formed neutron star at the center. The figure shows a snapshot of the entropy at $300 \mathrm{~ms}$ after core bounce and shock formation for a two-dimensional simulation of a $15 M_{\odot}$ star. The star was exploded by the neutrino-heating mechanism by chosing a suitable value of the neutrino luminosity from the nascent neutron star, which was replaced by an inner boundary condition 40,41]. The white line encompasses the region where radioactive nickel has been formed by nuclear burning in the shock-heated $\mathrm{Si}$ layer.

\subsection{Convective Overturn in the Neutrino-Heated Region}

Convective instabilities in the layers adjacent to the nascent neutron star are a natural consequence of the negative entropy gradient built up by the weakening of the prompt shock prior to its stagnation and by neutrino heating [4]. This was verified by two- and three-dimensional simulations [27, 47, 28, 20,28, 58, 32, 33, 45,. Figure 5 shows the entropy distribution between proto-neutron star and supernova shock about $300 \mathrm{~ms}$ after core bounce for one such calculation [40,41]. Although there is general agreement about the existence and the growth of hydrodynamic instabilities in the layer between the shock at $R_{\mathrm{s}}$ and the radius of maximum neutrino heating (which is just outside the gain radius, $R_{\mathrm{g}}$ ), the strength of the convective overturn and its importance for the success of the neutrino-heating mechanism are still a matter of debate.

Two-dimensional simulations with a spectrally averaged, flux-limited diffusion treatment of neutrino transport 28, 20], or with the neutrino luminosity being given as a free parameter at the inner boundary, which replaces the neutron star at the center [32,33], found successful explosions in cases where spherically symmetric models fail (Fig. 3). According to these simulations, the convective overturn in the neutrino-heated region has the following effects on the shock propagation. Heated matter from the region close to the gain radius rises outward and at the same time is exchanged with cool gas flowing down from the shock. Since the production reactions of neutrinos $\left(e^{ \pm}\right.$capture on nucleons and 
thermal processes) are very temperature sensitive, the expansion and cooling of rising plasma reduces the energy loss by the reemission of neutrinos. Moreover, the net energy deposition by neutrinos is enhanced as more cool material is exposed to the large neutrino fluxes near the gain radius (the radial dilution of the fluxes goes roughly as $1 / r^{2}$ ). Since hot matter moves towards the shock, the pressure behind the shock increases, an effect which pushes the shock farther out. This leads to a growth of the gain region and therefore also of the net energy transfer from neutrinos to the stellar gas, favoring an explosion.

The consequences of postshock convection are clearly visible from the results plotted in Fig. 3, where the explosion energy $E_{>0}$ as a function of time is shown for spherically symmetric and two-dimensional calculations of the same post-collapse model, but with different assumed neutrino luminosities from the proto-neutron star 32,33]. $E_{>0}$ is defined to include the sum of internal, kinetic, and gravitational energy for all zones where this sum is positive (the gravitational binding energies of stellar mantle and envelope and additional energy release from nuclear burning are not taken into account). For one-dimensional simulations with $\nu_{e}$ luminosities (and very similar $\bar{\nu}_{e}$ luminosities) below $1.9 \cdot 10^{52} \mathrm{erg} / \mathrm{s}$ explosions could not be obtained when the proto-neutron star was assumed static, and the threshold value of the luminosity was $2.2 \cdot 10^{52} \mathrm{erg} / \mathrm{s}$ when the neutron star was contracting. The supporting effects of convective overturn between the gain radius and the shock lead to explosions even below the critical value in spherical symmetry, and to a faster development of the explosion.

Simulations with a better description of the neutrino transport by a multienergy-group treatment of the neutrino diffusion [45], confirm the existence of such convective processes in the region of neutrino heating, but the associated effects are not strong enough to revive the stalled prompt supernova shock, although the outward motion of the shock is enhanced.

Fully self-consistent, multi-dimensional calculations, however, have not yet been done with a state-of-the-art Boltzmann neutrino transport, which has recently become applicable for spherically symmetric models (see Section 3 ). The current multi-dimensional simulations therefore demonstrate only the presence and potential importance of convection, but final conclusions on the viability of the neutrino-heating mechanism in the presence of postshock convection are not possible at the moment. A quantitatively meaningful description of the shock revival phase, however, requires an accurate description of the transport as well as a multi-dimensional approach.

\subsection{Is Neutrino-Driven Convection Crucial for an Explosion?}

The role of convective overturn for the development of an explosion becomes clearer by considering the three timescales of neutrino heating, $\tau_{\mathrm{ht}}$, advection of accreted matter through the gain layer into the cooling region and down to the neutron star (compare Fig. 1), $\tau_{\mathrm{ad}}$, and the timescale for the growth of convective instabilities, $\tau_{\mathrm{cv}}$. The evolution of the shock - accretion or explosion

- is determined by the relative size of these three timescales. Straightforward 


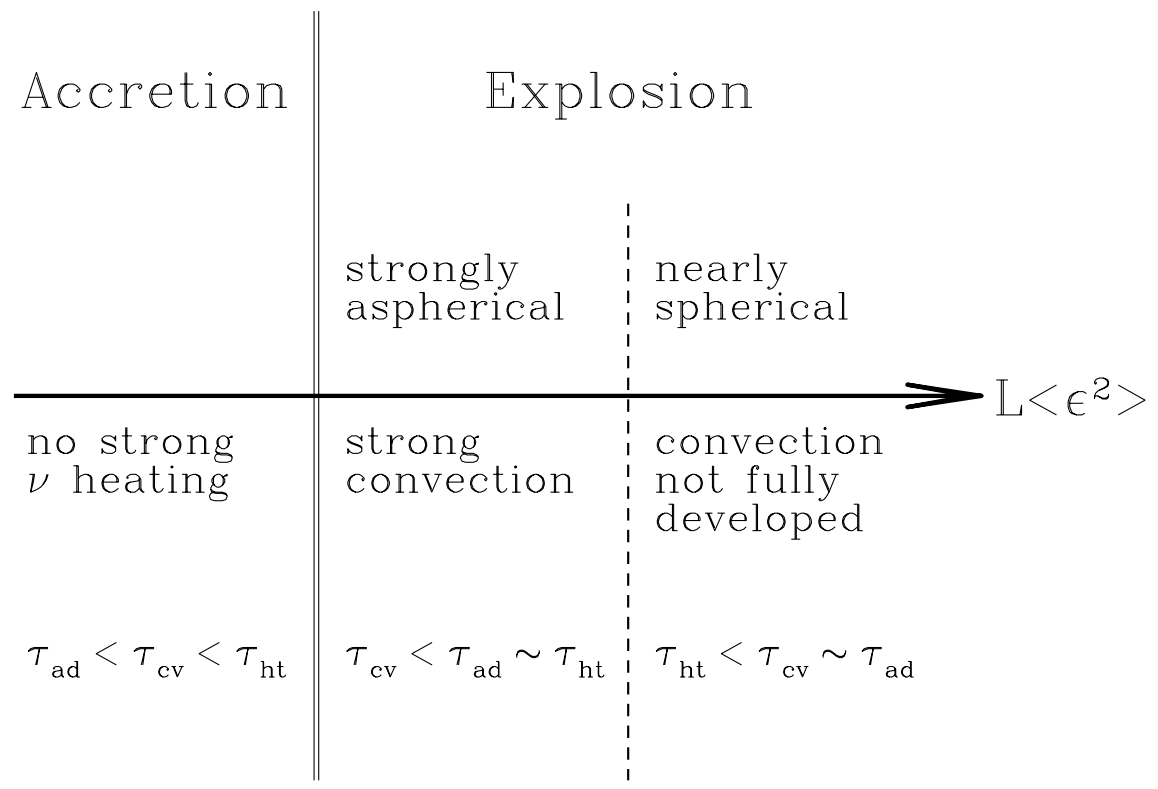

Fig. 6. Order scheme for the post-collapse dynamics in dependence of $L_{\nu}\left\langle\epsilon_{\nu}^{2}\right\rangle$, which determines the strength of the neutrino heating outside of the neutrinosphere. The destiny of the star - accretion or explosion - can be understood by the relative size of the timescales of neutrino heating, $\tau_{\mathrm{ht}}$, matter advection through the gain region onto the nascent neutron star, $\tau_{\mathrm{adv}}$, and growth of convective instabilities, $\tau_{\mathrm{cv}}$.

considerations show that they are of the same order and the destiny of the star is therefore a result of a tight competition between the different processes.

The heating timescale is estimated from the initial entropy per nucleon, $s_{\mathrm{i}}$, the critical entropy $s_{\mathrm{c}}$ (Eq. (10)), and the heating rate per nucleon (Eq. (ब) ) as

$$
\tau_{\mathrm{ht}} \approx \frac{s_{\mathrm{c}}-s_{\mathrm{i}}}{Q_{\nu}^{+} /\left(k_{\mathrm{B}} T\right)} \approx 45 \mathrm{~ms} \cdot \frac{s_{\mathrm{c}}-s_{\mathrm{i}}}{5 k_{\mathrm{B}} / N} \frac{R_{\mathrm{g}, 7}^{2}(T / 2 \mathrm{MeV}) f}{\left(L_{\nu} / 4 \cdot 10^{52} \mathrm{erg} / \mathrm{s}\right)\left\langle\epsilon_{\nu, 15}^{2}\right\rangle}
$$

for $L_{\nu}$ being the total luminosity of $\nu_{e}$ plus $\bar{\nu}_{e}$. With a postshock velocity of $u_{1}=u_{0} / \beta \approx(\gamma-1) \sqrt{G M / R_{\mathrm{s}}} /(\gamma+1)$ the advection timescale is

$$
\tau_{\mathrm{ad}} \approx \frac{R_{\mathrm{s}}-R_{\mathrm{g}}}{u_{1}} \approx 55 \mathrm{~ms} \cdot\left(1-\frac{R_{\mathrm{g}}}{R_{\mathrm{s}}}\right) \frac{R_{\mathrm{s}, 200}^{3 / 2}}{\sqrt{M_{1}}}
$$

where the gain radius can be determined as

$$
R_{\mathrm{g}, 7} \cong 0.9 T_{\mathrm{s}}^{3 / 2} R_{\mathrm{s}, 200}^{3 / 2} f^{1 / 4}\left(\frac{L_{\nu}}{4 \cdot 10^{52} \mathrm{erg} / \mathrm{s}}\right)^{-1 / 4}\left\langle\epsilon_{\nu, 15}^{2}\right\rangle^{-1 / 4}
$$


from the requirement that the heating rate, Eq. (4), is equal to the cooling rate per nucleon, $Q_{\nu}^{-} \approx 2.3 T^{6} \mathrm{MeV} /(N \cdot \mathrm{s})$, when $R_{\mathrm{s}, 200}$ is the shock radius in units of $200 \mathrm{~km}, Y_{n}+Y_{p} \approx 1$ is assumed, and use is made of the power-law behavior of the temperature according to $T(r) \approx T_{\mathrm{S}}\left(R_{\mathrm{S}} / r\right)$, with $T_{\mathrm{s}}$ being the postshock temperature in $\mathrm{MeV}$. The growth timescale of convective instabilities in the neutrino-heated region depends on the gradients of entropy and lepton number through the growth rate of Ledoux convection, $\sigma_{\mathrm{L}}$ :

$$
\begin{aligned}
\tau_{\mathrm{cv}} \approx \frac{\ln (100)}{\sigma_{\mathrm{L}}} & \approx 4.6\left\{\frac{g}{\rho}\left[\left(\frac{\partial \rho}{\partial s}\right)_{Y_{e}, P} \frac{\mathrm{d} s}{\mathrm{~d} r}+\left(\frac{\partial \rho}{\partial Y_{e}}\right)_{s, P} \frac{\mathrm{d} Y_{e}}{\mathrm{~d} r}\right]\right\}^{-1 / 2} \\
& \sim 20 \mathrm{~ms} \cdot\left(\frac{R_{\mathrm{s}}}{R_{\mathrm{g}}}-1\right)^{1 / 2} \frac{R_{\mathrm{g}, 7}^{3 / 2}}{\sqrt{M_{1}}}
\end{aligned}
$$

The numerical value was obtained with the gravitational acceleration $g=G M / R_{\mathrm{g}}^{2}$, $(\partial \rho / \partial s)_{P} \sim-\rho / s$, and $\mathrm{d} s / \mathrm{d} r \sim-\frac{1}{2} s /\left(R_{\mathrm{s}}-R_{\mathrm{g}}\right)$. The term proportional to the gradient of $Y_{e}$ was assumed to be negligible. $\tau_{\mathrm{cv}}$ of Eq. (17) is sensitive to the detailed conditions between gain radius (close to which $s$ develops a maximum) and the shock. The neutrino heating timescale is shorter for larger values of the neutrino luminosity $L_{\nu}$ and mean squared neutrino energy $\left\langle\epsilon_{\nu}^{2}\right\rangle$. All three timescales, $\tau_{\mathrm{ht}}, \tau_{\mathrm{ad}}$ and $\tau_{\mathrm{cv}}$, decrease roughly in the same way with smaller gain radius or shock position.

In order to be a crucial help for the explosion, convective overturn in the neutrino-heated region must develop on a sufficiently short timescale. This happens only in a rather narrow window of $L_{\nu}\left\langle\epsilon_{\nu}^{2}\right\rangle$ where $\tau_{\mathrm{cv}}<\tau_{\mathrm{ad}} \sim \tau_{\mathrm{ht}}$ (Fig. (6). For smaller neutrino luminosities the heating is too weak to create a sufficiently large entropy maximum, and rapid convective motions cannot develop before the accreted gas is advected through the gain radius $\left(\tau_{\mathrm{ad}}<\tau_{\mathrm{cv}}<\tau_{\mathrm{ht}}\right)$. In this case neither with nor without convective processes energetic explosions can occur (Fig. 3). For larger neutrino luminosities the neutrino heating is so strong, and the heating timescale correspondingly short $\left(\tau_{\mathrm{ht}}<\tau_{\mathrm{cv}} \sim \tau_{\mathrm{ad}}\right)$, that expansion of the postshock layers has set in before the convective activity reaches a significant level. In this case convective overturn is an unavoidable side-effect of the neutrino heating behind the shock, but is not necessary for starting the explosion.

The parametric studies performed by Janka and Müller [32.33] support this discussion, which helps one understanding the seemingly discrepant results obtained by different groups.

\subsection{Nucleosynthesis and Mixing Instabilities}

Besides increasing the efficiency of neutrino energy deposition, convection in the postshock layer has an important influence also on the nucleosynthesis and distribution of radioactive elements. In particular, nickel is not produced by silicon burning in a spherical shell, but is concentrated in dense clumps and pockets 
Fig. 7. Snapshot from a two-dimensional simulation of the explosion of a blue supergiant star with 15 solar masses at a time 1170 seconds after the stellar core has collapsed to a neutron star 41. In the left half of the figure the density is shown in a region with a radius of about 2.2 million kilometers, in the right half three color images of the mass densities of radioactive nickel (red and pink), silicon (green, light blue, whitish) and oxygen (deep blue) are superposed. One can see that the ejecta of the explosion are inhomogeneous and anisotropic, and the original onion-shell structure of the exploding star was shredded. Nickel is concentrated in dense, fast-moving clumps along the extended filaments seen in the left plot.
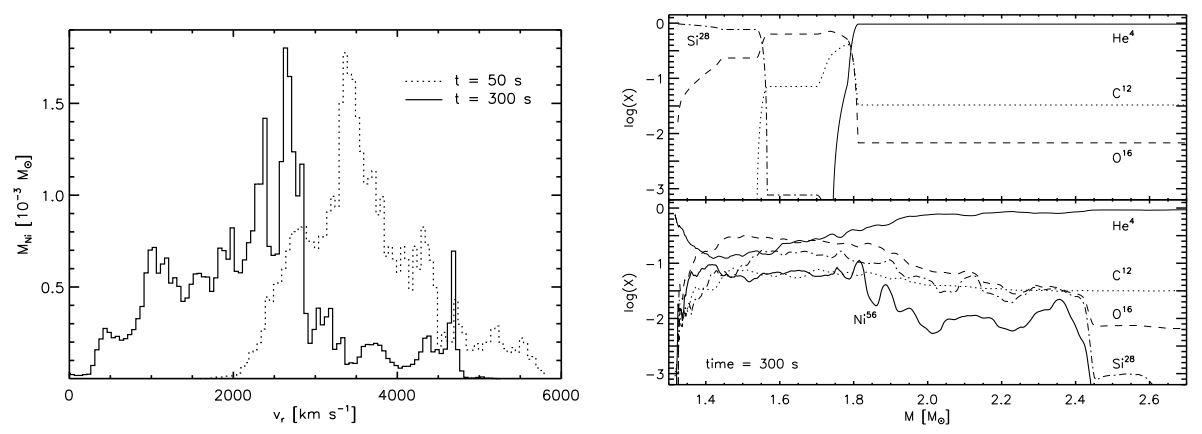

Fig. 8. Left: Distribution of ${ }^{56} \mathrm{Ni}$ vs. radial velocity at $t=50 \mathrm{~s}$ and $t=300 \mathrm{~s}$ after core bounce. Right: Initial composition of the star exterior to the iron core (top) and composition $300 \mathrm{~s}$ after core bounce (bottom). C, O, Si, and the newly synthesized Ni have been mixed beyond the inner half of the helium core, and He has been carried inward.

between rising bubbles of neutrino-heated matter in the expanding postshock layer (Fig. 5).

The further evolution of the shock until it breaks out of the stellar surface hours later, was recently followed by using adaptive mesh refinement techniques 40,41]. These allow for a dynamic adjustment of the computational grid such that small structures can be treated with high resolution, while the whole computation covers a huge volume.

A few seconds after its formation, the shock has passed the silicon and oxygen layers and propagates through the helium shell of the star. The initial anisotropies have been compressed into a narrow shell, from which new instabilities start to grow. Rayleigh-Taylor mushrooms penetrate into the helium layer 
and carry $\mathrm{O}, \mathrm{Si}$ and $\mathrm{Ni}$ farther out, while He sinks in. Within minutes, long, dense filaments reach far into the helium shell, associated with them fast-moving knots that contain dominant contributions of different heavy elements from the deeper layers (Fig. Đ). Nickel, silicon and oxygen move through helium with velocities up to several $1000 \mathrm{~km} / \mathrm{s}$ (Fig. 8).

These simulations show that the hydrodynamic instabilities which occur in the first second of the explosion, do not only play a role during the phases of shock rejuvenation and nickel formation. They act also as seed perturbations for the instabilities at the composition interfaces of the progenitor, which finally destroy the onion-shell structure of the pre-collapse star.

The results are in good agreement with observations of mixing and anisotropies in many Type Ib,c supernovae. In case of Supernova 1987A, a Type II explosion of a massive star which has retained its hydrogen envelope, the observed high nickel velocities in the hydrogen envelope cannot be explained by the models. The nickel clumps are strongly decelerated at the $\mathrm{He} / \mathrm{H}$ interface, where they enter a dense helium "wall" which builds up after the passage of the shock. The dissipation of the kinetic energy of the clumps does not allow nickel to penetrate into the hydrogen layer with high velocities.

\section{Neutron Star Formation}

Convective energy transport inside the newly formed neutron star can increase the neutrino luminosities considerably [17]. This can be crucial for energizing the stalled supernova shock [64,65].

Convection in the neutron star can be driven by gradients of the entropy and/or proton (electron lepton number) fraction in the nuclear medium 22. The type of instability which grows most rapidly, e.g., doubly diffusive neutron-finger convection [64,65] or Ledoux convection [17] or quasi-Ledoux convection [36.38], may be a matter of the properties of the nuclear equation of state, which determines the magnitudes and signs of the thermodynamic derivatives [13. It is also sensitive to the gradients that develop, and thus may depend on the details of the treatment of neutrino transport in the dense interior of the star.

Convection below the neutrinosphere seems to be disfavored during the very early post-bounce evolution by the currently most elaborate supernova models 14, 15, 44, but can develop deeper inside the nascent neutron star on a longer timescale ( $\gtrsim 100 \mathrm{~ms}$ after bounce) and can encompass the whole star within seconds [17, 38, 36].

Negative lepton number and entropy gradients have been seen in several onedimensional (spherically symmetric) simulations of the neutrino-cooling phase of nascent neutron stars [16, 17, 37,59] (see also Fig. 9) and have suggested the existence of regions which are potentially unstable against Ledoux convection. Recent calculations [50] with improved neutrino opacities of the nuclear medium, which were described consistently with the employed equation of state, confirm principal aspects of previous simulations, in particular the existence of Ledouxunstable layers in the neutron star. 

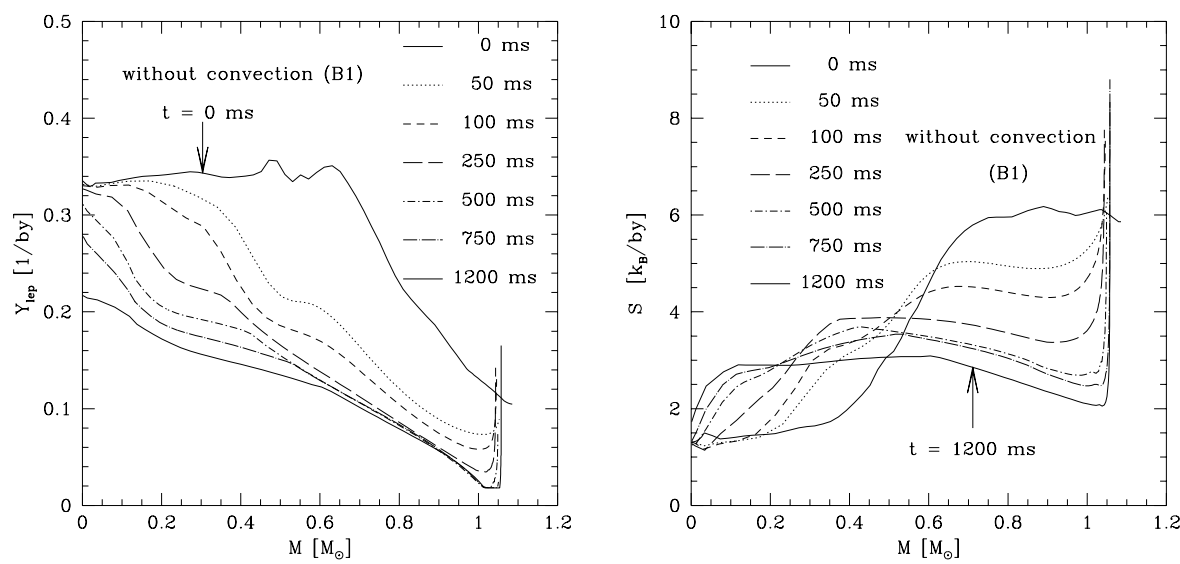

Fig. 9. Profiles of the lepton fraction $Y_{\text {lep }}=n_{\text {lep }} / n_{b}$ (left) and of the entropy per nucleon, $s$, (right) as functions of enclosed (baryonic) mass for different times in a one-dimensional simulation of the neutrino cooling of a $\sim 1.1 M_{\odot}$ proto-neutron star. Negative gradients of lepton number and entropy suggest potentially convectively unstable regions. Time is (roughly) measured from core bounce.

\subsection{Convection inside the Nascent Neutron Star}

Two-dimensional, hydrodynamical simulations were performed for the neutrinocooling phase of a $\sim 1.1 M_{\odot}$ proto-neutron star that formed in the core collapse of a $15 M_{\odot}$ star [38, 36]. The models followed the evolution for a period of more than 1.2 seconds. They demonstrate the development of convection and its importance for the cooling and deleptonization of the neutron star.

The simulations were carried out with the hydrodynamics code Prometheus. A general relativistic 1D gravitational potential with Newtonian corrections for asphericities was used, $\Phi \equiv \Phi_{1 \mathrm{D}}^{\mathrm{GR}}+\left(\Phi_{2 \mathrm{D}}^{\mathrm{N}}-\Phi_{1 \mathrm{D}}^{\mathrm{N}}\right)$, and a flux-limited (equilibrium) neutrino diffusion scheme was applied for each angular bin separately (" $1 \frac{1}{2} \mathrm{D}$ ").

The simulations showed that convectively unstable surface-near regions (i.e., around the neutrinosphere and below an initial density of about $10^{12} \mathrm{~g} / \mathrm{cm}^{3}$ ) exist only for a short period of a few ten milliseconds after bounce, in agreement with the findings by other groups [14,15, 44. Due to a flat entropy profile and a negative lepton number gradient, convection, however, also starts in a layer deeper inside the star, between an enclosed mass of $0.7 M_{\odot}$ and $0.9 M_{\odot}$, at densities above several $10^{12} \mathrm{~g} / \mathrm{cm}^{3}$. From there the convective region digs into the star and reaches the center after about one second (Figs. 10, 11, and 13). Convective velocities as high as $5 \cdot 10^{8} \mathrm{~cm} / \mathrm{s}$ were found (about $10-20 \%$ of the local sound speed), corresponding to kinetic energies of up to $1-2 \cdot 10^{50} \mathrm{erg}$ (Fig. 11). Because of these high velocities and rather flat entropy and composition profiles in the star (Fig. 10), the overshooting region is large (see Fig. 13). The same is true for undershooting during the first $\sim 100 \mathrm{~ms}$ after bounce. Sound waves and 

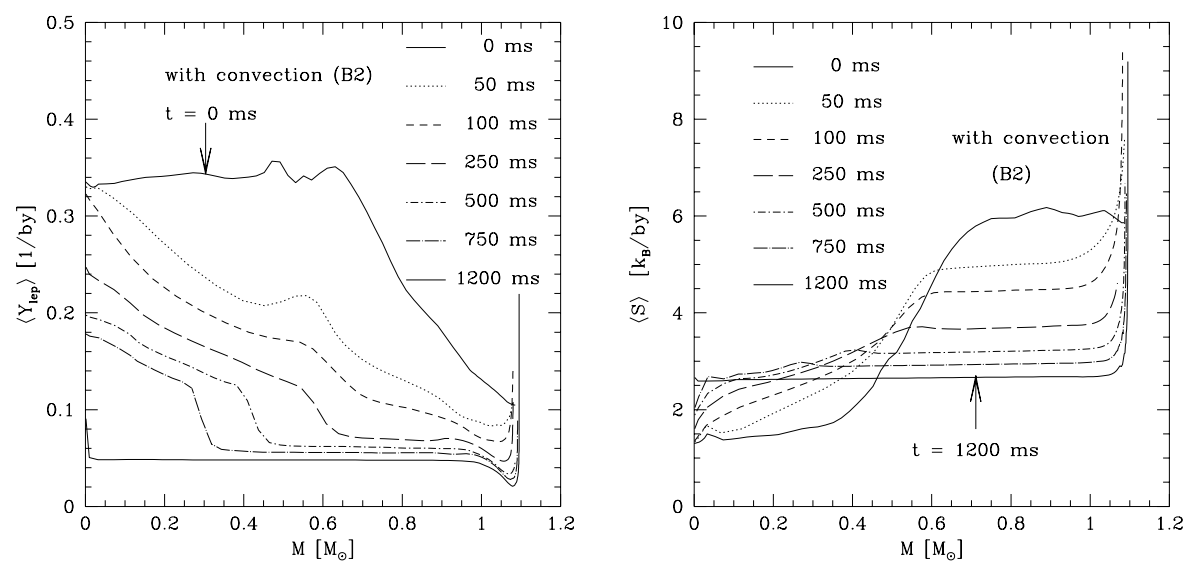

Fig. 10. Same as Fig. 9, but for a two-dimensional, hydrodynamical simulation which allowed to follow the development of convection. The plots show angularly averaged quantities in the $\sim 1.1 M_{\odot}$ proto-neutron star. In regions with convective activity the gradients of $Y_{\text {lep }}$ and $s$ are flattened. The convective layer encompasses an increasingly larger part of the star.

Fig. 11. Absolute values of the convective velocity in the proto-neutron star for two instants (about $0.5 \mathrm{~s}$ (left) and 1s (right) after core bounce) as obtained in a twodimensional, hydrodynamical simulation. The arrows indicate the direction of the velocity field. Note that the neutron star has contracted from a radius of about $60 \mathrm{~km}$ initially to little more than $20 \mathrm{~km}$. The growth of the convective region can be seen. Typical velocities of the convective motions are several $10^{8} \mathrm{~cm} / \mathrm{s}$.

perturbations are generated in the layers above and interior to the convection zone.

The coherence lengths of convective structures are of the order of $20-40$ degrees (in 2D!) (see Fig. 11) and coherence times are around $10 \mathrm{~ms}$, which corresponds to only one or two overturns. The convective pattern is therefore very time-dependent and nonstationary. Convective motions lead to considerable variations of the composition. The lepton fraction (and thus the abundance of protons) shows relative fluctuations of several $10 \%$. The entropy differences in 


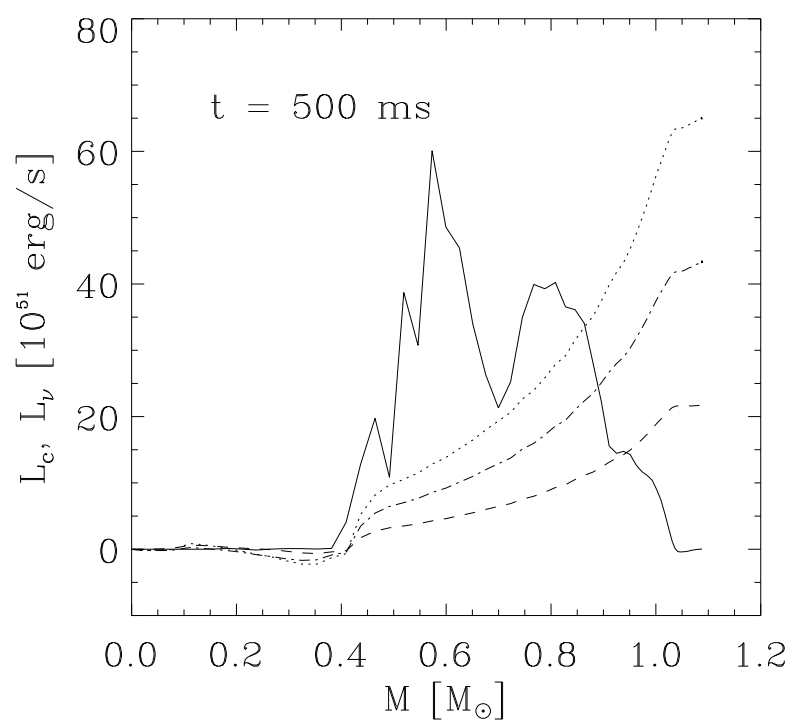

Fig. 12. Convective "luminosity" (solid line) and neutrino luminosities (dashed: $L_{\nu_{e}}+$ $L_{\bar{\nu}_{e}}$, dash-dotted: $L_{\nu_{\mu}}+L_{\bar{\nu}_{\mu}}+L_{\nu_{\tau}}+L_{\bar{\nu}_{\tau}}$, dotted: total) as functions of enclosed baryonic mass for the two-dimensional proto-neutron star simulation about $500 \mathrm{~ms}$ after core bounce.

rising and sinking convective bubbles are much smaller, only a few per cent, while temperature and density fluctuations are typically less than one per cent.

The energy transport in the neutron star is dominated by neutrino diffusion near the center, whereas convective transport plays the major role in a thick intermediate layer where the convective activity is strongest. Radiative transport takes over again when the neutrino mean free path becomes large near the surface of the star (Fig. 12). But even in the convective layer the convective energy flux is only a few times larger than the diffusive flux. This means that neutrino diffusion is not negligibly small in the convective region. This fact has important consequences for the driving mechanism of the convection.

\subsection{Driving Force of Convection}

The convective activity in the neutron star cannot be explained by, and considered as ideal Ledoux convection. Applying the Ledoux criterion for local instability,

$$
C_{\mathrm{L}}(r, \theta)=\frac{\rho}{g} \sigma_{\mathrm{L}}^{2}=\left(\frac{\partial \rho}{\partial s}\right)_{Y_{\mathrm{lep}}, P} \frac{\mathrm{d} s}{\mathrm{~d} r}+\left(\frac{\partial \rho}{\partial Y_{\mathrm{lep}}}\right)_{s, P} \frac{\mathrm{d} Y_{\text {lep }}}{\mathrm{d} r}>0
$$

with $\sigma_{\mathrm{L}}$ from Eq. (17) and $Y_{e}$ replaced by the total lepton fraction $Y_{\text {lep }}$ in the neutrino-opaque interior of the neutron star (for reasons of simplicity, $\nabla s$ was 
Fig. 13. Left: Convectively unstable region (corresponding to negative values of the displayed quantity $\omega_{\mathrm{QL}}^{2}=-(g / \rho) C_{\mathrm{QL}}$ with $C_{\mathrm{QL}}$ from Eq. (19)) about $500 \mathrm{~ms}$ after bounce according to the Quasi-Ledoux criterion which includes non-adiabatic and lepton-transport effects by neutrino diffusion. Right: Layer of Quasi-Ledoux convective instability (blue) as function of time for a two-dimensional simulation. The angleaveraged criterion $C_{\mathrm{QL}}^{1 \mathrm{D}}(r) \equiv \min _{\theta}\left(C_{\mathrm{QL}}(r, \theta)\right)>0$ with $C_{\mathrm{QL}}(r, \theta)$ from Eq. $\left.\sqrt{19}\right)$ is plotted. The dotted area is outside of the computed star, green denotes stable layers where over- and undershooting causes lateral velocities with angularly averaged absolute values of $\left\langle\left|v_{\theta}\right|\right\rangle>10^{7} \mathrm{~cm} \mathrm{~s}^{-1}$, and white are convectively "quiet" regions of the star.

replaced by $\mathrm{d} s / \mathrm{d} r$ and $\nabla Y_{\text {lep }}$ by $\left.\mathrm{d} Y_{\text {lep }} / \mathrm{d} r\right)$, one finds that the convecting region should actually be stable, despite of slightly negative entropy and lepton number gradients. In fact, below a critical value of the lepton fraction (e.g., $Y_{\text {lep }, \mathrm{c}}=0.148$ for $\rho=10^{13} \mathrm{~g} / \mathrm{cm}^{3}$ and $T=10.7 \mathrm{MeV}$ ) the thermodynamical derivative $\left(\partial \rho / \partial Y_{\text {lep }}\right)_{s, P}$ changes sign and becomes positive because of nuclear and Coulomb forces in the high-density equation of state [13]. Therefore negative lepton number gradients should stabilize against convection in this regime. However, an idealized assumption of Ledoux convection is not fulfilled in the situations considered here: Because of neutrino diffusion, energy exchange and, in particular, lepton number exchange between convective elements and their surroundings are not negligible. Taking the neutrino transport effects on $Y_{\text {lep }}$ into account in a modified "Quasi-Ledoux criterion" [36],

$$
C_{\mathrm{QL}}(r, \theta) \equiv\left(\frac{\partial \rho}{\partial s}\right)_{\left\langle Y_{\text {lep }}\right\rangle,\langle P\rangle} \frac{\mathrm{d}\langle s\rangle}{\mathrm{d} r}+\left(\frac{\partial \rho}{\partial Y_{\text {lep }}}\right)_{\langle s\rangle,\langle P\rangle}\left(\frac{\mathrm{d}\left\langle Y_{\text {lep }}\right\rangle}{\mathrm{d} r}-\beta_{\text {lep }} \frac{\mathrm{d} Y_{\text {lep }}}{\mathrm{d} r}\right)>0
$$

one determines instability exactly where the two-dimensional simulation reveals convective activity. In Eq. (19) the quantities $\left\langle Y_{\text {lep }}\right\rangle$ and $\langle s\rangle$ mean averages over the polar angles $\theta$, and local gradients have to be distinguished from gradients of angle-averaged quantities which describe the stellar background. The term $\beta_{\text {lep }}\left(\mathrm{d} Y_{\text {lep }} / \mathrm{d} r\right)$ with the empirically determined value $\beta_{\text {lep }} \approx 1$ accounts for the change of the lepton concentration along the path of a rising fluid element due to neutrino diffusion. Figure 13 shows that about half a second after core bounce strong, driving forces for convection occur in a narrow ring between 9 and $10 \mathrm{~km}$, where a steep negative gradient of the lepton fraction exists (see Fig. 10). Farther out, convective instability is detected only in finger-like structures of rising, high$Y_{\text {lep gas. }}$ 
Fig. 14. Absolute value of the gas velocity in a convecting, rotating proto-neutron star about $750 \mathrm{~ms}$ after bounce (left). Convection is suppressed near the rotation axis (vertical) and develops strongly only near the equatorial plane where a flat distribution of the specific angular momentum $j_{z}$ (right) has formed.

\subsection{Accretion and Rotation}

In other two-dimensional models, post-bounce mass accretion and rotation of the forming neutron star were included. Accretion causes stronger convection with larger velocities in a more extended region. This can be explained by the steepening of lepton number and entropy gradients and the increase of the gravitational potential energy when additional matter is added onto the neutron star.

Rotation has very interesting consequences, e.g., leads to a suppression of convective motions near the rotation axis because of a stabilizing stratification of the specific angular momentum (see Fig. 14), an effect which can be understood by applying the (first) Solberg-Høiland criterion for instabilities in rotating, selfgravitating bodies 61]:

$$
C_{\mathrm{SH}}(r, \theta) \equiv \frac{1}{x^{3}} \frac{\mathrm{d} j_{z}^{2}}{\mathrm{~d} x}+\frac{\boldsymbol{a}}{\rho}\left[\left(\frac{\partial \rho}{\partial s}\right)_{Y_{\mathrm{lep}}, P} \nabla s+\left(\frac{\partial \rho}{\partial Y_{\mathrm{lep}}}\right)_{s, P} \nabla Y_{\mathrm{lep}}\right]<0
$$

Here, $j_{z}$ is the specific angular momentum of a fluid element, which is conserved for axially symmetric configurations, $x$ is the distance from the rotation axis, and in case of rotational equilibrium $\boldsymbol{a}$ is the sum of gravitational and centrifugal accelerations, $\boldsymbol{a}=\nabla P / \rho$. Changes of the lepton number in rising or sinking convective elements due to neutrino diffusion were neglected in Eq. (20). Ledoux (or Quasi-Ledoux) convection can only develop where the first term is not too positive. In Fig. 14 fully developed convective motion is therefore constrained to a zone of nearly constant $j_{z}$ close to the equatorial plane. At higher latitude the convective velocities are much smaller, and narrow, elongated convective cells aligned with cylindrical regions of $j_{z}=$ const parallel to the rotation axis are visible.

The rotation pattern displayed in Fig. 14 is highly differential with a rotation period of $7.3 \mathrm{~ms}$ at $x=22 \mathrm{~km}$ and of $1.6 \mathrm{~ms}$ at $x=0.6 \mathrm{~km}$. It has self-consistently developed under the influence of neutrino transport and convection when the neutron star had contracted from an initial radius of about $60 \mathrm{~km}$ (with a surface 

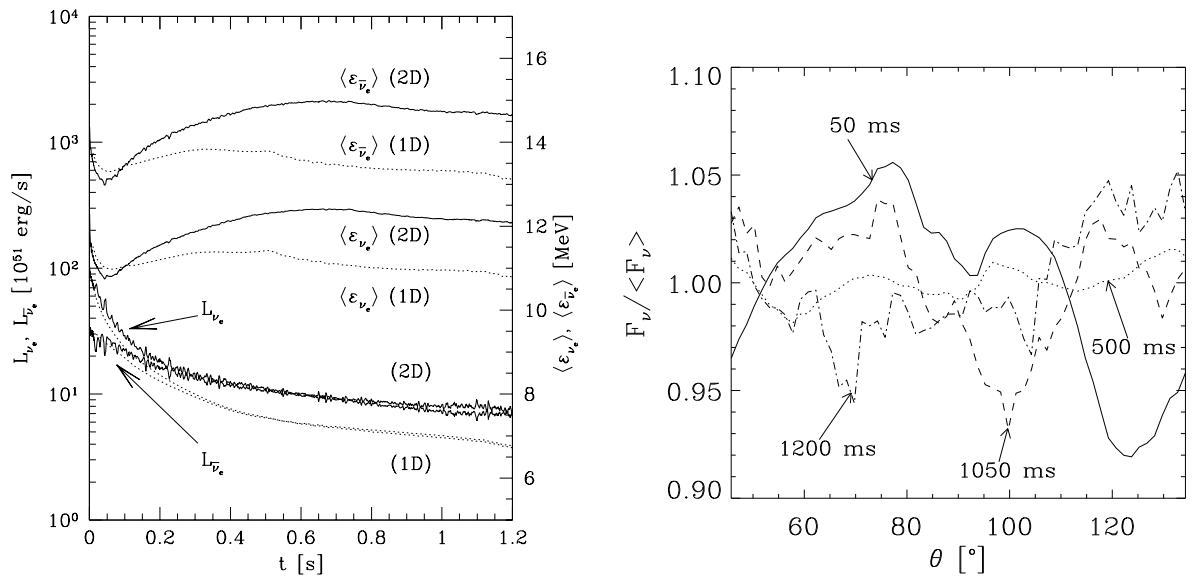

Fig. 15. Left: Luminosities $L_{\nu}(t)$ and mean energies $\left\langle\epsilon_{\nu}\right\rangle(t)$ of $\nu_{e}$ and $\bar{\nu}_{e}$ for a $1.1 M_{\odot}$ proto-neutron star without ("1D"; dotted) and with convection ("2D"; solid). Right: Angular variations of the neutrino flux at different times for the $2 \mathrm{D}$ simulation.

rotation period of $55 \mathrm{~ms}$ at the equator and a rotation period of $\sim 5 \mathrm{~ms}$ near the center) to a final radius of approximately $22 \mathrm{~km}$. Due to the differential nature of the rotation, the ratio of rotational kinetic energy to the gravitational potential energy of the star is only $0.78 \%$ in the beginning and a few per cent at the end after about $1 \mathrm{~s}$ of evolution.

\subsection{Consequences of Proto-Neutron Star Convection}

Convection inside the proto-neutron star can raise the neutrino luminosities within a few hundred ms after core bounce (Fig. 15). In the considered collapsed core of a $15 M_{\odot}$ star, $L_{\nu_{e}}$ and $L_{\bar{\nu}_{e}}$ increase by up to $50 \%$ and the mean neutrino energies by about $15 \%$ at times later than $200-300 \mathrm{~ms}$ post bounce. This favors neutrino-driven explosions on timescales of a few hundred milliseconds after shock formation. Also, the deleptonization of the nascent neutron star is strongly accelerated, raising the $\nu_{e}$ luminosities relative to the $\bar{\nu}_{e}$ luminosities during this time. This helps to increase the electron fraction $Y_{e}$ in the neutrino-heated ejecta and might solve the overproduction problem of $N=50$ nuclei during the early epochs of the explosion [38. In case of rotation, the effects of convection on the neutrino emission depend on the direction. Since strong convection occurs only close to the equatorial plane, the neutrino fluxes are convectively enhanced there, while they are essentially unchanged near the poles.

Anisotropic mass motions due to convection in the neutron star lead to gravitational wave emission and anisotropic radiation of neutrinos. The angular variations of the neutrino flux found in the 2D simulations are of the order of $5-10 \%$ (Fig. 15). With the typical size of the convective cells and the short coherence times of the convective structures, the global anisotropy of the neutrino emission 


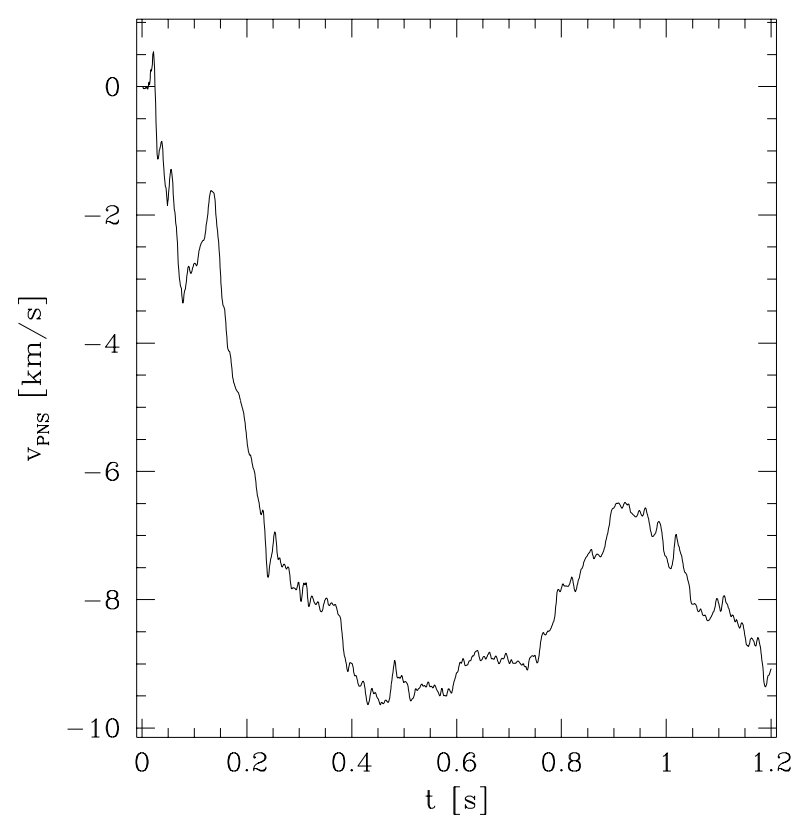

Fig. 16. Kick velocity of the neutron star as a function of time, caused by the anisotropic emission of neutrinos due to convection. The two-dimensional simulation was done with a polar grid from 0 to $\pi$.

from the cooling proto-neutron star is very small. This implies a kick velocity of the nascent neutron star due to anisotropic neutrino emission of only $\sim 10 \mathrm{~km} / \mathrm{s}$ in a $2 \mathrm{D}$ simulation (Fig. 16). Because the convective elements are likely to become even smaller in 3D, kick velocities of $300 \mathrm{~km} / \mathrm{s}$ or even more, as observed for many pulsars, can definitely not be explained by convectively perturbed neutrino emission.

\subsection{Neutrino Opacities in Nuclear Matter and Neutron Star Convection}

Another important issue of interest are the neutrino opacities in the dense and hot nuclear medium of the nascent neutron star. In current supernova models, the description of neutrino-nucleon interactions is incomplete because the standard approximations assume isolated and infinitely massive nucleons [62. Therefore effects like the fermion phase space blocking of the nucleons, the reduction of the effective nucleon mass by momentum-dependent nuclear interactions in the dense plasma, and nucleon thermal motions and recoil are either neglected completely or approximated in a more or less reliable manner [9,16]. These effects have been recognized to be important [57, 34,51,55] for calculations of the neutrino luminosities and spectra, but still await careful inclusion in supernova codes. For 

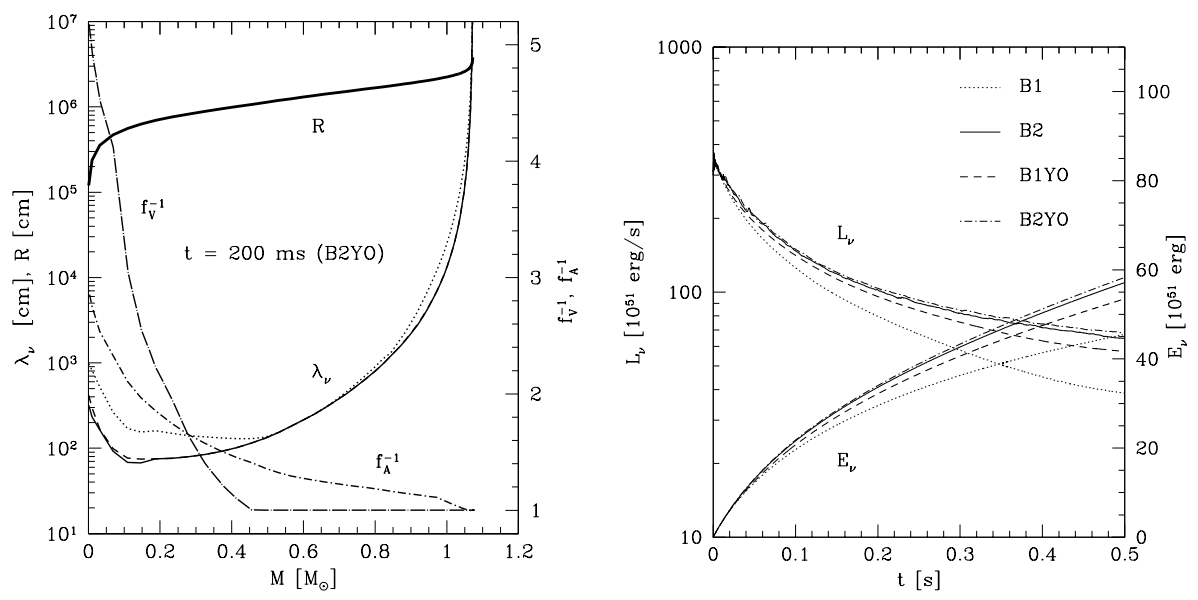

Fig. 17. Left: Thermal averages of the neutrino mean free paths for $\nu_{e}$ absorption (dotted line), $\nu_{e}$ scattering (solid line), and muon and tau neutrino scattering (dashed line), respectively, according to the standard description of the neutrino opacities. $M$ is the baryonic mass enclosed by the radial coordinate $R$ (bold solid line) of the newly formed neutron star about $200 \mathrm{~ms}$ after core bounce. Also shown are the factors $f_{\mathrm{A}}^{-1}$ and $f_{\mathrm{V}}^{-1}$ (dash-dotted lines) which give a measure of the increase of the neutrino mean free paths caused by a suppression of the axial-vector and vector current contributions to the neutrino opacities due to in-medium effects 68 (Yamada, personal communication). Right: Total neutrino luminosities, $L_{\nu}$, and integrated energy loss, $E_{\nu}$, as functions of time for spherically symmetric models without convection (B1 and B1YO) and twodimensional models with convection (B2 and B2YO). Models B1 and B2 were computed with standard neutrino opacities whereas in B1YO and B2YO in-medium suppression of the neutrino opacities was included [68] (Yamada, personal communication).

this purpose a consistent description of nuclear equation of state and neutrinomatter interactions is desirable.

Many-body (spatial) correlations due to strong interactions [56, 29, $19,55,68$ and multiple-scattering effects by spin-dependent forces between nucleons (temporal spin-density correlations) [52,26] are of particular interest, because they lead to a reduction of the neutrino opacities in the newly formed neutron star and are associated with additional modes of energy transfer between neutrinos and the nuclear medium.

A reduction of the neutrino opacities implies larger neutrino mean free paths and thus increases the neutrino luminosities. (Fig. 17 and Refs. 39,50,19]). The neutrino diffusion is accelerated most strongly in the very dense core of the nascent neutron star. Convection in the intermediate region between core and outer layers turns out not to be suppressed, but is still the fastest mode of energy transport. Therefore reduced neutrino opacities as well as convective energy transport are important, but the combined effects do not appreciably change the convectively enhanced neutrino emission (Fig. 17, right) 335. 


\section{Summary}

Supernova explosions of massive stars are an important phenomenon for applying nuclear and particle physics, in particular neutrino physics. The processes going on in the extremely dense and hot core of the exploding star are accessible to direct measurements only through neutrinos or gravitational waves. Empirical information about the events that cause the explosion and accompany the formation of a neutron star, however, can also be deduced from observable characteristics of supernovae, for example their explosion energy or the amount and distribution of radioactive nuclei, and from the properties of neutron stars.

Theoretical models need to establish the link between the core physics and these observables. In the past years it has been recognized that hydrodynamical instabilities and mixing processes on large scales play an important role within the core as well as in the outer layers of the exploding star. Convection can change the cooling of the nascent neutron star, supports the revival of the stalled shock by neutrino heating, and destroys the onion-shell structure of the progenitor star. Multi-dimensional calculations are therefore necessary to understand why and how supernovae explode, and to make predictions for their observable consequences.

Spherically symmetric simulations, Newtonian and general relativistic, with the most advanced treatment of neutrino transport by solving the Boltzmann equation, do not produce explosions. This emphasizes the importance of convection, but may also point to physics still missing in the models. One such weakness of current simulations is an overly simplified description of neutrino interactions with nucleons in the nuclear medium of the neutron star. A kinematically correct treatment of these reactions, taking into account nucleon thermal motions, recoil and fermi blocking, needs only a technical step, but a better understanding of the effects of nucleon correlations and their consistent treatment with the equation of state requires theoretical progress.

The neutrino-heating mechanism, although the favored explanation for the explosion, is still controversial, both because of the status of modeling and because of observations which seem hard to explain. Although significant progress has been made, multi-dimensional simulations with an accurate and reliable handling of neutrino transport and an up-to-date treatment of the input physics are still missing, and definite conclusions can therefore not be drawn at the moment.

Acknowledgements. H.-Th. Janka thanks E. Müller and W. Keil for many years of fruitful and enjoyable collaboration. This work was supported by the Sonderforschungsbereich 375 on "Astroparticle Physics" of the Deutsche Forschungsgemeinschaft.

\section{References}

1. E.N. Alexeyev, et al.: Phys. Lett. B205, 209 (1988) R.M. Bionta, et al.: Phys. Rev. Lett. 58, 1494 (1987) K. Hirata, et al.: Phys. Rev. Lett. 58, 1490 (1987) 
2. W. Baade, F. Zwicky: Phys. Rev. 45, 138 (1934)

3. E. Baron, J. Cooperstein: Astrophys. J. 353, 597 (1990)

4. H.A. Bethe: Rev. Mod. Phys. 62, 801 (1990)

5. H.A. Bethe: Astrophys. J. 412, 192 (1993)

6. H.A. Bethe: Astrophys. J. 449, 714 (1995)

7. H.A. Bethe: Astrophys. J. 490, 765 (1997)

8. H.A. Bethe, J.R. Wilson: Astrophys. J. 295, 14 (1985)

9. S.W. Bruenn: Astrophys. J. Suppl. 58, 771 (1985)

10. S.W. Bruenn: Phys. Rev. Lett. 59, 938 (1987)

11. S.W. Bruenn: Astrophys. J. 340, 955 (1989) Astrophys. J. 341, 385 (1989)

12. S.W. Bruenn: 'Numerical Simulations of Core Collapse Supernovae'. In: Nuclear Physics in the Universe, ed. by M.W. Guidry, M.R. Strayer (IOP, Bristol 1993) p. 31

13. S.W. Bruenn, T. Dineva: Astrophys. J. 458, L71 (1996)

14. S.W. Bruenn, A. Mezzacappa: Astrophys. J. 433, L45 (1994)

15. S.W. Bruenn, A. Mezzacappa, T. Dineva: Phys. Rep. 256, 69 (1995)

16. A. Burrows, J.M. Lattimer: Astrophys. J. 307, 178 (1986)

17. A. Burrows: Astrophys. J. 318, L57 (1987) A. Burrows, J.M. Lattimer: Phys. Rep. 163, 51 (1988)

18. A. Burrows, J. Goshy: Astrophys. J. 416, L75 (1993)

19. A. Burrows, R.F. Sawyer: Phys. Rev. C58, 554 (1998) A. Burrows, R.F. Sawyer: Phys. Rev. C59, 510 (1999)

20. A. Burrows, J. Hayes, B.A. Fryxell: Astrophys. J. 450, 830 (1995)

21. S.A. Colgate, R.H. White: Astrophys. J. 143, 626 (1966)

22. R.I. Epstein: Mon. Not. R. Astron. Soc. 188, 305 (1979)

23. C.L. Fryer: Astrophys. J. 522, 413 (1999)

24. C.L. Fryer, A. Heger: Astrophys. J. 541, 1033 (2000)

25. C.L. Fryer, V. Kalogera: Astrophys. J., submitted (astro-ph/9911312)

26. S. Hannestad, G. Raffelt: Astrophys. J. 507, 339 (1998)

27. M. Herant, W. Benz, S.A. Colgate: Astrophys. J. 395, 642 (1992)

28. M. Herant, W. Benz, W.R. Hix, C.L. Fryer, S.A. Colgate: Astrophys. J. 435, 339 (1994)

29. C.J. Horowitz, K. Wehrberger: Phys. Lett. B266, 236 (1991) C.J. Horowitz, K. Wehrberger: Nucl. Phys. A531, 665 (1991)

30. K. Iwamoto, et al.: Nature 395, 672 (1998) M. Turatto, et al.: Astrophys. J. 534, L57 (2000) K. Iwamoto, et al.: Astrophys. J. 534, 660 (2000)

31. H.-Th. Janka: Astron. Astrophys., submitted (astro-ph/0008432)

32. H.-Th. Janka, E. Müller: Astrophys. J. 448, L109 (1995)

33. H.-Th. Janka, E. Müller: Astron. Astrophys. 306, 167 (1996)

34. H.-Th. Janka, W. Keil, G. Raffelt, D. Seckel: Phys. Rev. Lett. 76, 2621 (1996)

35. H.-Th. Janka, W. Keil, S. Yamada: in preparation (2001)

36. W. Keil: Konvektive Instabilitäten in entstehenden Neutronensternen. PhD Thesis, Technical University, Munich (1997)

37. W. Keil, H.-Th. Janka: Astron. Astrophys. 296, 145 (1995)

38. W. Keil, H.-Th. Janka, E. Müller: Astrophys. J. 473, L111 (1996)

39. W. Keil, H.-Th. Janka, G. Raffelt: Phys. Rev. D51, 6635 (1995)

40. K. Kifonidis: Nucleosynthesis and Hydrodynamic Instabilities in Core Collapse Supernovae. PhD Thesis, Technical University, Munich (2001)

41. K. Kifonidis, T. Plewa, H.-Th. Janka, E. Müller: Astrophys. J. 531, L123 (2000)

42. M. Liebendörfer, A. Mezzacappa, F.-K. Tielemann, O.E.B. Messer, W.R. Hix, S.W. Bruenn: Phys. Rev. D, submitted (astro-ph/0006418) 
43. O.E.B. Messer, A. Mezzacappa, S.W. Bruenn, M.W. Guidry: Astrophys. J. 507, $353(1998)$

44. A. Mezzacappa, A.C. Calder, S.W. Bruenn, J.M. Blondin, M.W. Guidry, M.R. Strayer, A.S. Umar: Astrophys. J. 493, 848 (1998)

45. A. Mezzacappa, A.C. Calder, S.W. Bruenn, J.M. Blondin, M.W. Guidry, M.R. Strayer, A.S. Umar: Astrophys. J. 495, 911 (1998)

46. A. Mezzacappa, M. Liebendörfer, O.E.B. Messer, W.R. Hix, F.-K. Tielemann, S.W. Bruenn: Phys. Rev. Lett., submitted (astro-ph/0005366)

47. D.S. Miller, J.R. Wilson, R.W. Mayle: Astrophys. J. 415, 278 (1993)

48. J.A. Miralles, J.A. Pons, V.A. Urpin: Astrophys. J. 543, 1001 (2000)

49. E.S. Myra, S.A. Bludman: Astrophys. J. 340, 384 (1989)

50. J.A. Pons, S. Reddy, M. Prakash, J.M. Lattimer, J.A. Miralles: Astrophys. J. 513, 780 (1999)

51. M. Prakash, et al.: Phys. Rep. 280, 1 (1997)

52. G.G. Raffelt, D. Seckel: Phys. Rev. D52, 1780 (1995) G.G. Raffelt, D. Seckel, G. Sigl: Phys. Rev. D54, 2784 (1996)

53. M. Rampp: Radiation Hydrodynamics with Neutrinos: Stellar Core Collapse and the Explosion Mechanism of Type II Supernovae. PhD Thesis, Technical University, Munich (2000)

54. M. Rampp, H.-Th. Janka: Astrophys. J. 539, L33 (2000)

55. S. Reddy, M. Prakash M.: Astrophys. J. 478, 689 (1997) S. Reddy, M. Prakash, J.M. Lattimer: Phys. Rev. D58, 013009 (1998)

56. R.F. Sawyer: Phys. Rev. C40, 865 (1989)

57. P.J. Schinder: Astrophys. J. Suppl. 74, 249 (1990)

58. T. Shimizu, S. Yamada, K. Sato: Astrophys. J. 432, L119 (1994)

59. K. Sumiyoshi, H. Suzuki, H. Toki: Astron. Astrophys. 303, 475 (1995)

60. F.D. Swesty, J.M. Lattimer, E.S. Myra: Astrophys. J. 425, 195 (1994)

61. J.-L. Tassoul: Theory of Rotating Stars. (Princeton Univ. Press, Princeton 1978)

62. D.L. Tubbs, D.N. Schramm: Astrophys. J. 201, 467 (1975)

63. J.R. Wilson: 'Supernovae and Post-Collapse Behavior'. In: Numerical Astrophysics, ed. by J.M. Centrella, J.M. LeBlanc, R.L. Bowers, J.A. Wheeler (Jones and Bartlett, Boston 1985) p. 422

64. J.R. Wilson, R. Mayle: Phys. Rep. 163, 63 (1988)

65. J.R. Wilson, R. Mayle: Phys. Rep. 227, 97 (1993)

66. J.R. Wilson, R. Mayle, S.E. Woosley, T. Weaver: Ann. NY Acad. Sci. 470, 267 (1986)

67. S.E. Woosley, T.A. Weaver: Astrophys. J. Suppl. 101, 181 (1995) F.X. Timmes, S.E. Woosley, T.A. Weaver: Astrophys. J. 457, 834 (1996)

68. S. Yamada: Nucl. Phys. A662, 219 (2000) S. Yamada, H. Toki: Phys. Rev. C61, $5803(2000)$

69. S. Yamada, H.-Th. Janka, H. Suzuki: Astron. Astrophys. 344, 533 (1999) 
This figure "Janka-fig1.png" is available in "png" format from: http://arxiv.org/ps/astro-ph/0103015v1 
This figure "Janka-fig4.png" is available in "png" format from: http://arxiv.org/ps/astro-ph/0103015v1 
This figure "Janka-fig5.jpeg" is available in "jpeg" format from: http://arxiv.org/ps/astro-ph/0103015v1 
This figure "Janka-fig7.jpeg" is available in "jpeg" format from: http://arxiv.org/ps/astro-ph/0103015v1 
This figure "Janka-fig11.png" is available in "png" format from: http://arxiv.org/ps/astro-ph/0103015v1 
This figure "Janka-fig13.png" is available in "png" format from: http://arxiv.org/ps/astro-ph/0103015v1 
This figure "Janka-fig14.png" is available in "png" format from: http://arxiv.org/ps/astro-ph/0103015v1 